\title{
PALEOENVIRONMENTAL AND PALEOCLIMATE INFERENCES FROM GASTROPOD \\ ASSEMBLAGES IN LAST GLACIAL MAXIMUM LOESS: ILLINOIS, INDIANA, AND KENTUCKY
}

David A. Grimley ${ }^{1}$, Caitlin Lebel ${ }^{2}$, Sarah Dendy ${ }^{3}$, Jessica L. Conroy ${ }^{3}$, Henry M. Loope 4
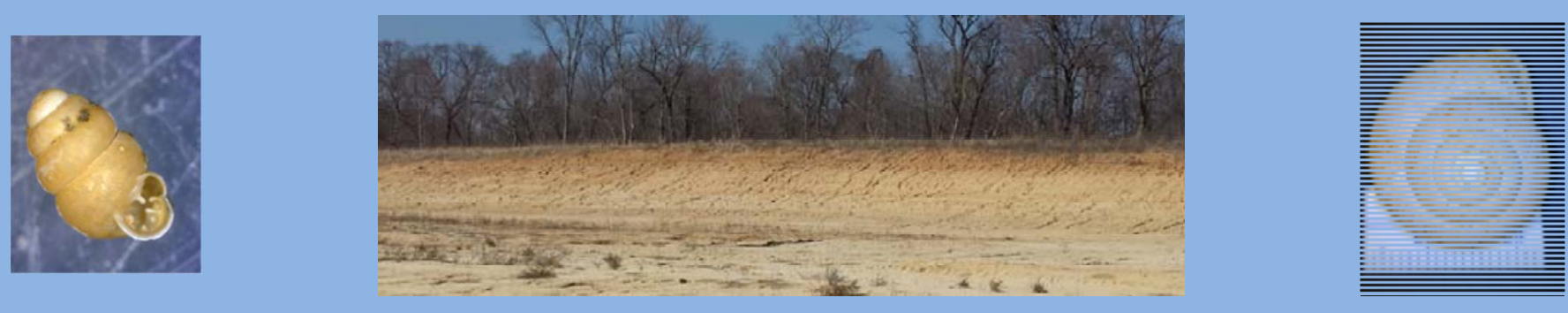

${ }^{2}$ Department of Geology Augustana College
${ }^{3}$ Department of Geology University of Illinois
${ }^{4}$ Indiana Geological and Water Survey Indiana University 


\section{Acknowledgements}

-- Ron Counts and Scott Wannigan aided with sampling and provided shells from the Rocks Section, Kentucky

-- Shannon Geil assisted with loess sampling at the Demazenod Section

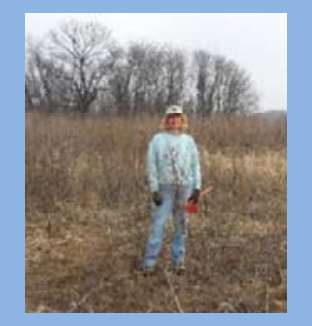

-- Andy Nash analyzed gastropods from New Cottonwood School and Thomas Quarry

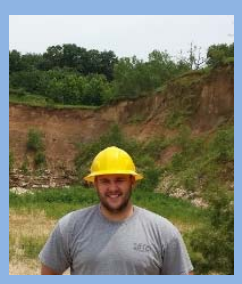

-- Catherine Nield (UIUC) prepared shells for isotopes from the Demazenod Section

-- NSF award GLD-1637481 provided funding for this project "Chronologies and mechanisms of last glacial loess deposition in the Central Lowlands of North America"

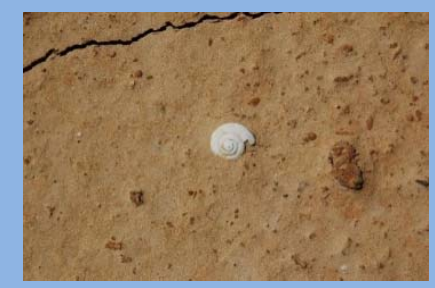




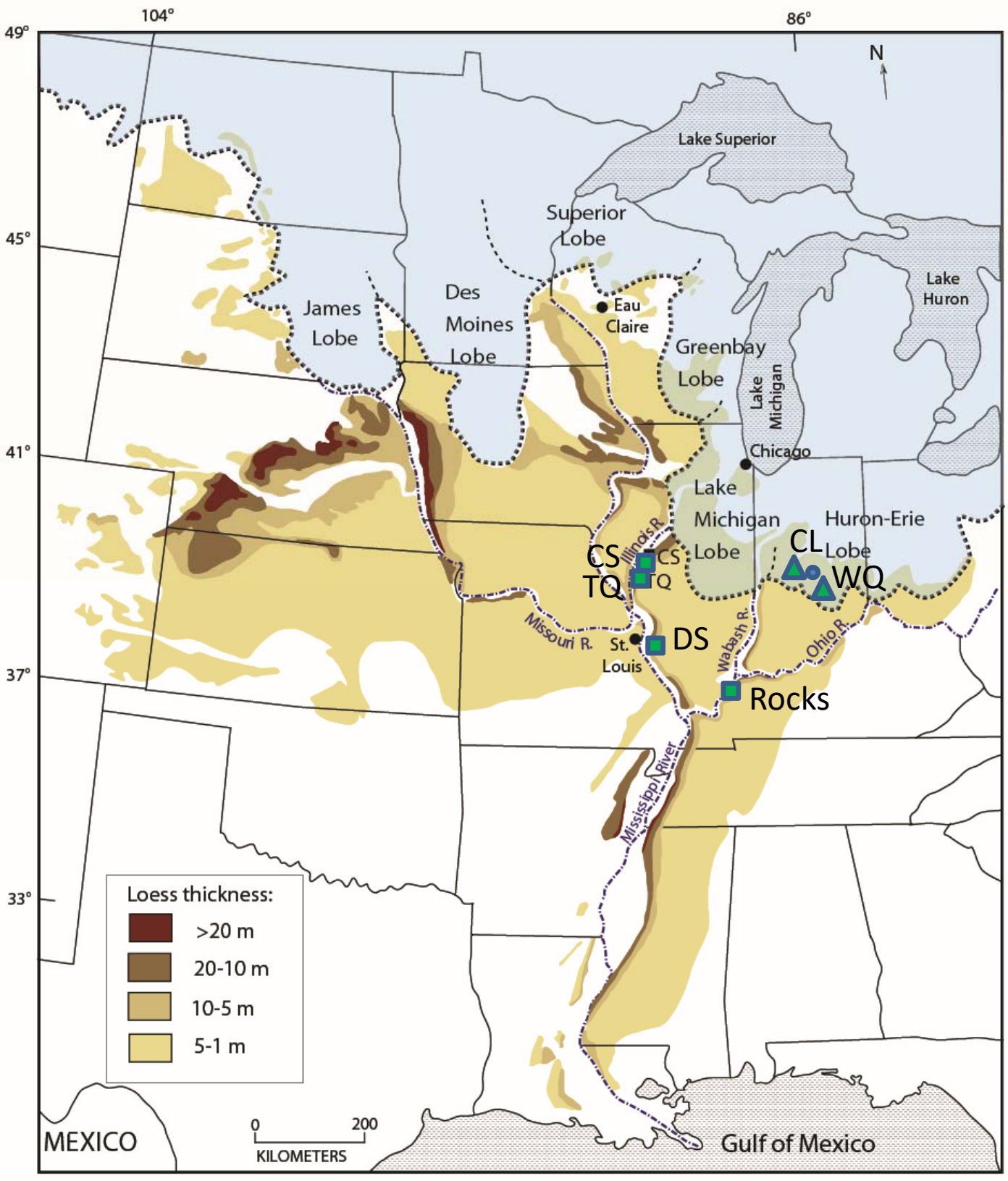

\section{Location of Study Area}

- loess sites in proximal uplands along the Illinois, Mississippi, and Ohio River Valleys

(glacigenic loess related to glacial meltwater valleys)

$\Delta \quad$ resedimented loess (silt) between last glacial tills in central Indiana 


\section{Research Objectives}

- high resolution chronology (using Succineidae; Pigati et al., 2015)

- paleoecological estimate; based on terrestrial gastropod assemblage

- paleoclimate estimate; based on gastropod assemblage and isotopes

- spatial and temporal variations in LGM ecology and climate; comparison to modern analogs
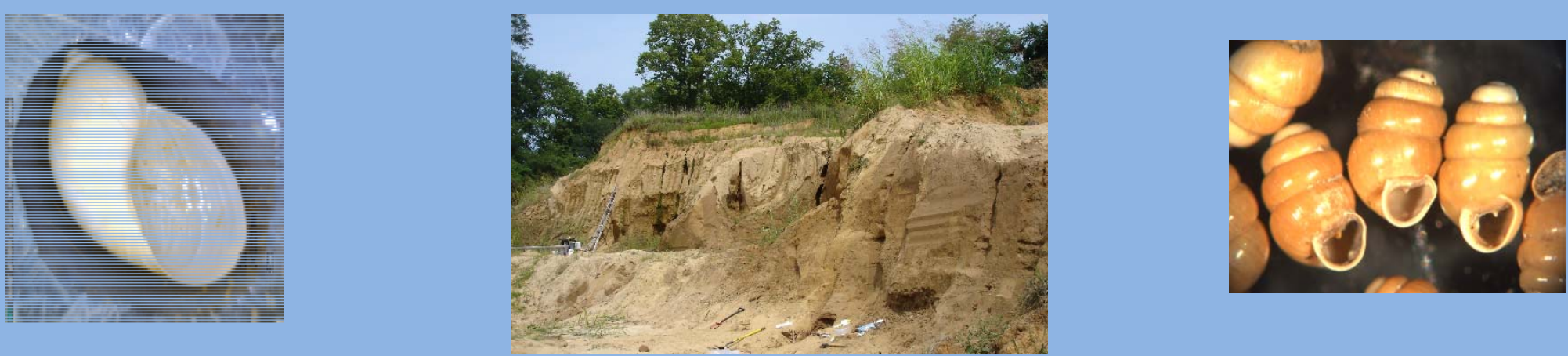


\section{New Cottonwood School Section}
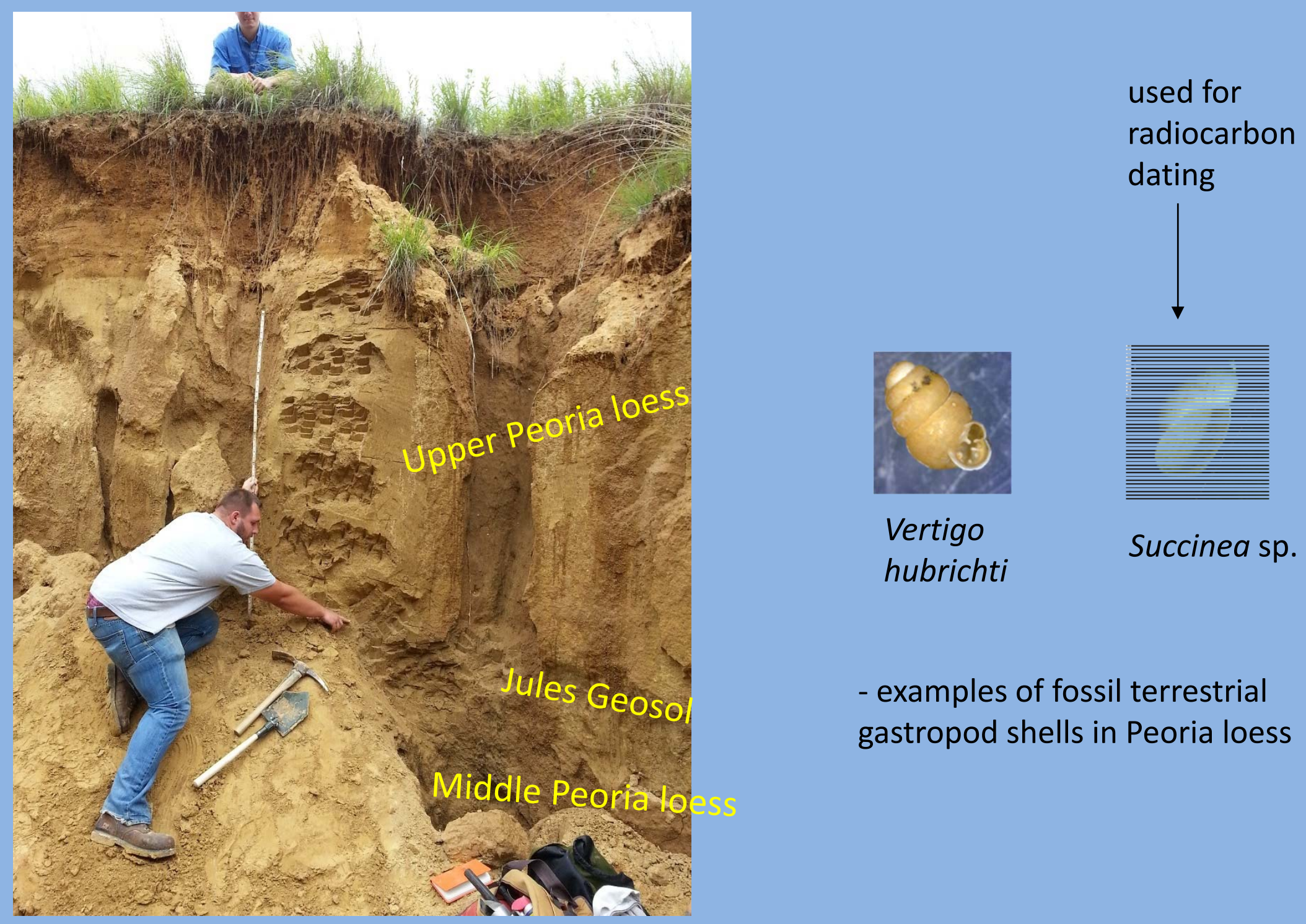

on uneroded flat upland

- examples of fossil terrestrial gastropod shells in Peoria loess 


\section{Thomas Quarry Sections}

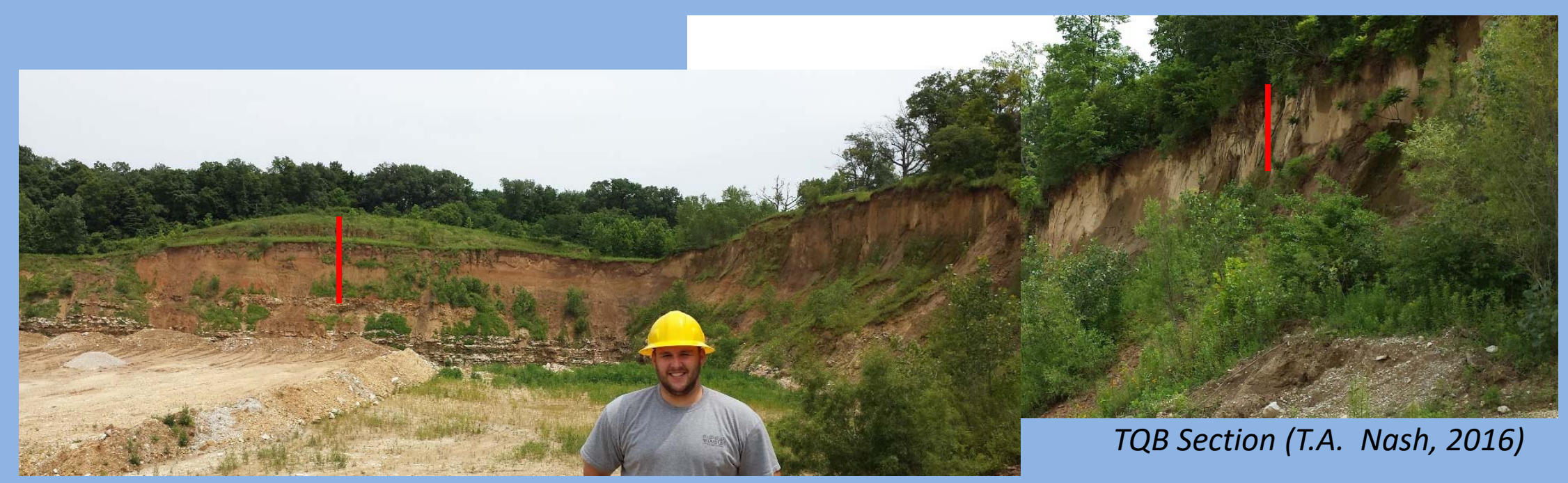

original TQ section (Grimley et al., 1998)

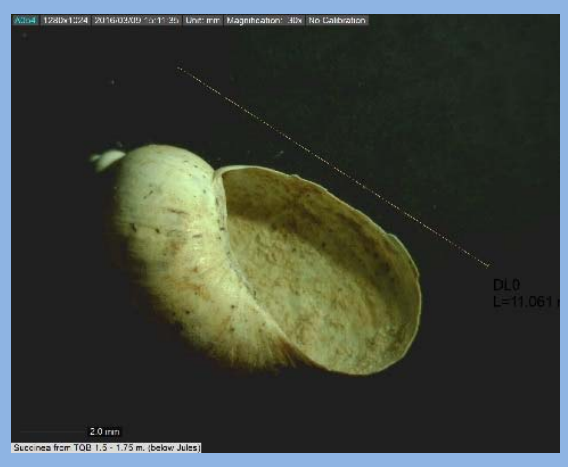

Succinea $\mathrm{sp}$. TQB 1.5-1.75

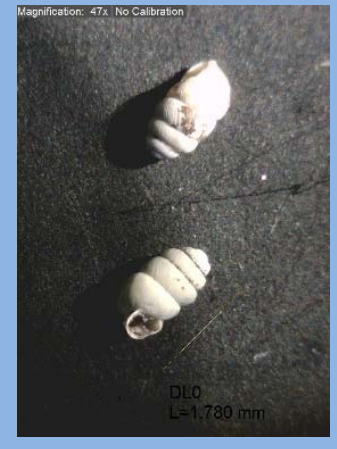

V. hubrichti; TQB 0.5 to $0.75 \mathrm{~m}$

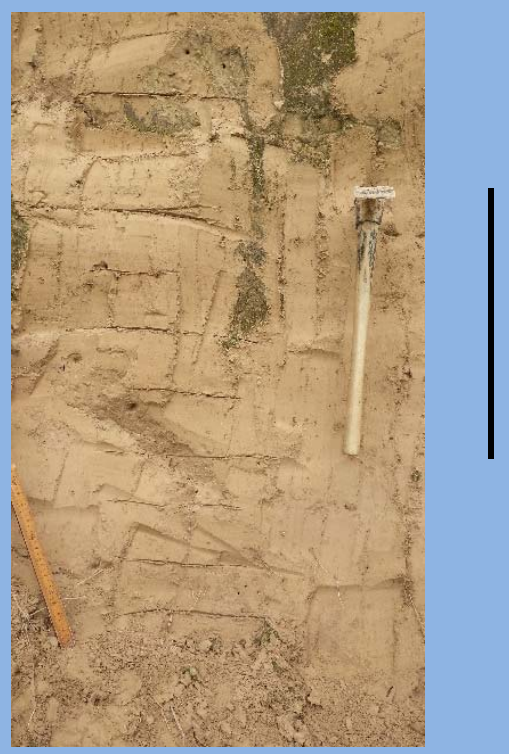

lower Peoria Silt; TQA 


\section{Chronologic Model (mainly Succineidae)}

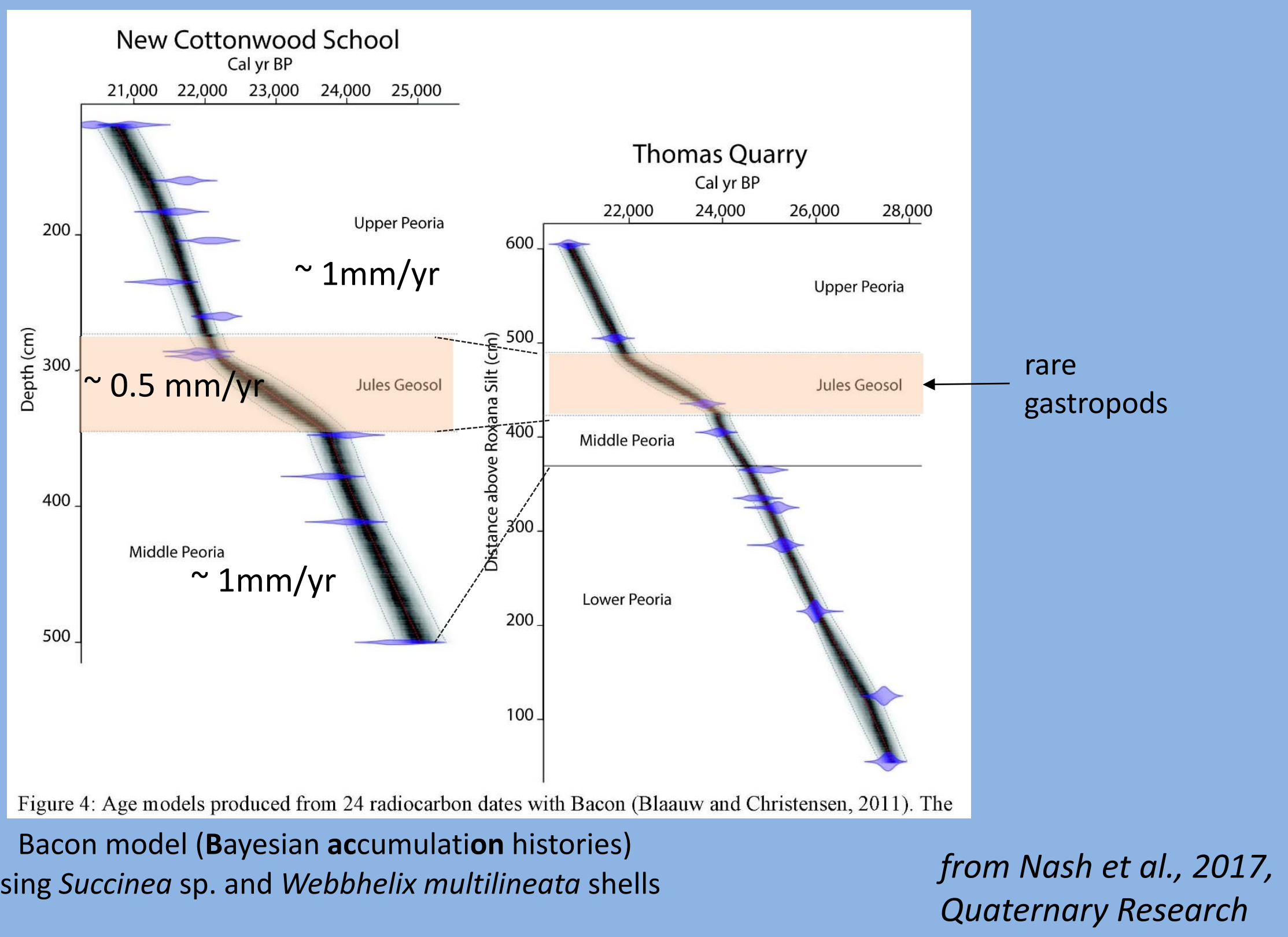




\section{Illinois Valley loess: gastropod assemblage}

- all terrestrial species

- live mainly in woodlands

- cool-cold boreal climate
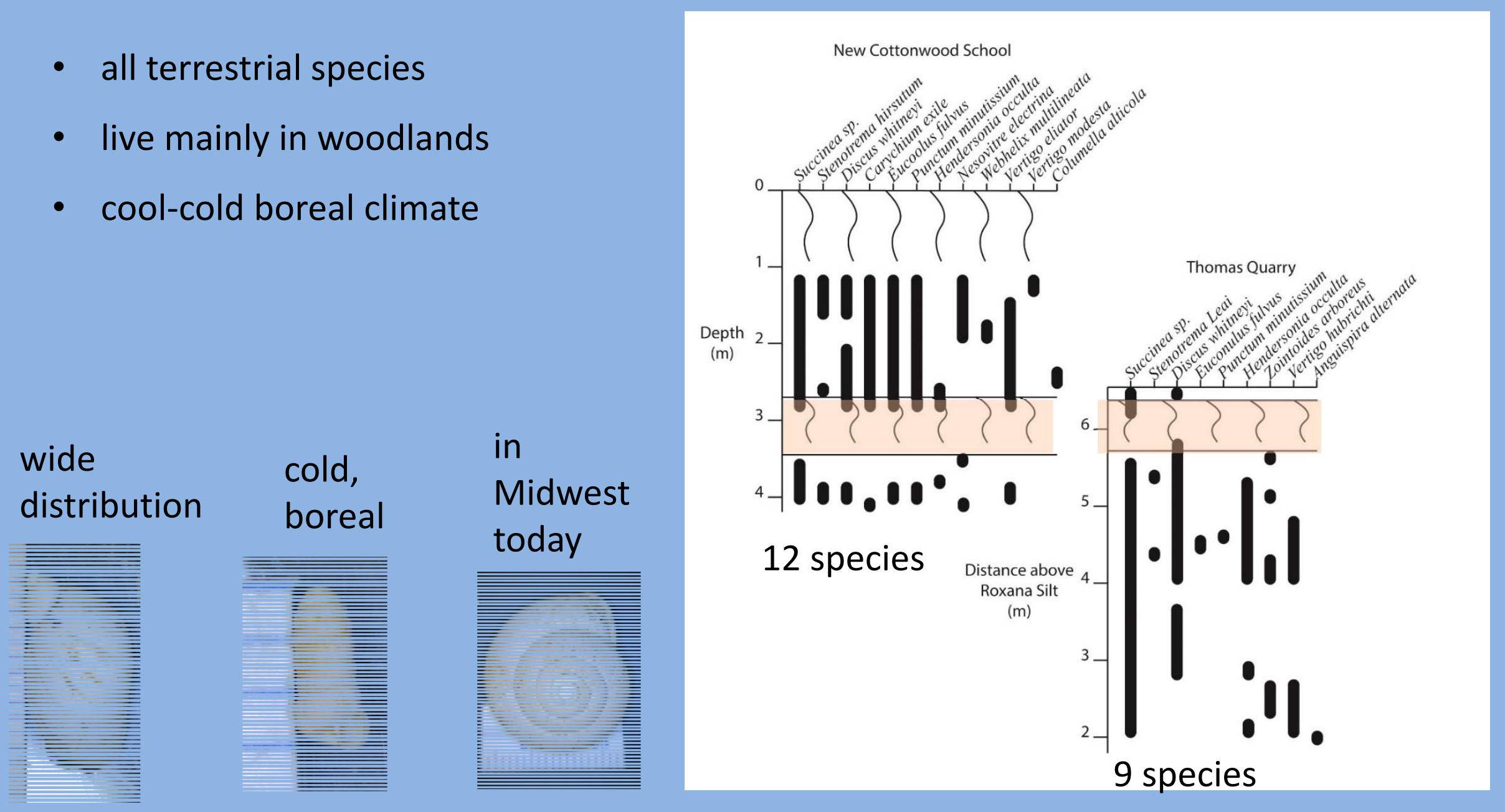

Succinea Columella Webbhelix

sp. alticola multilineata

from Nash et al., 2017, Quaternary Research 


\section{Gastropod Paleoecology}

Vertigo eliator:

1 to $1.5 \mathrm{~m}$ depth NSCA

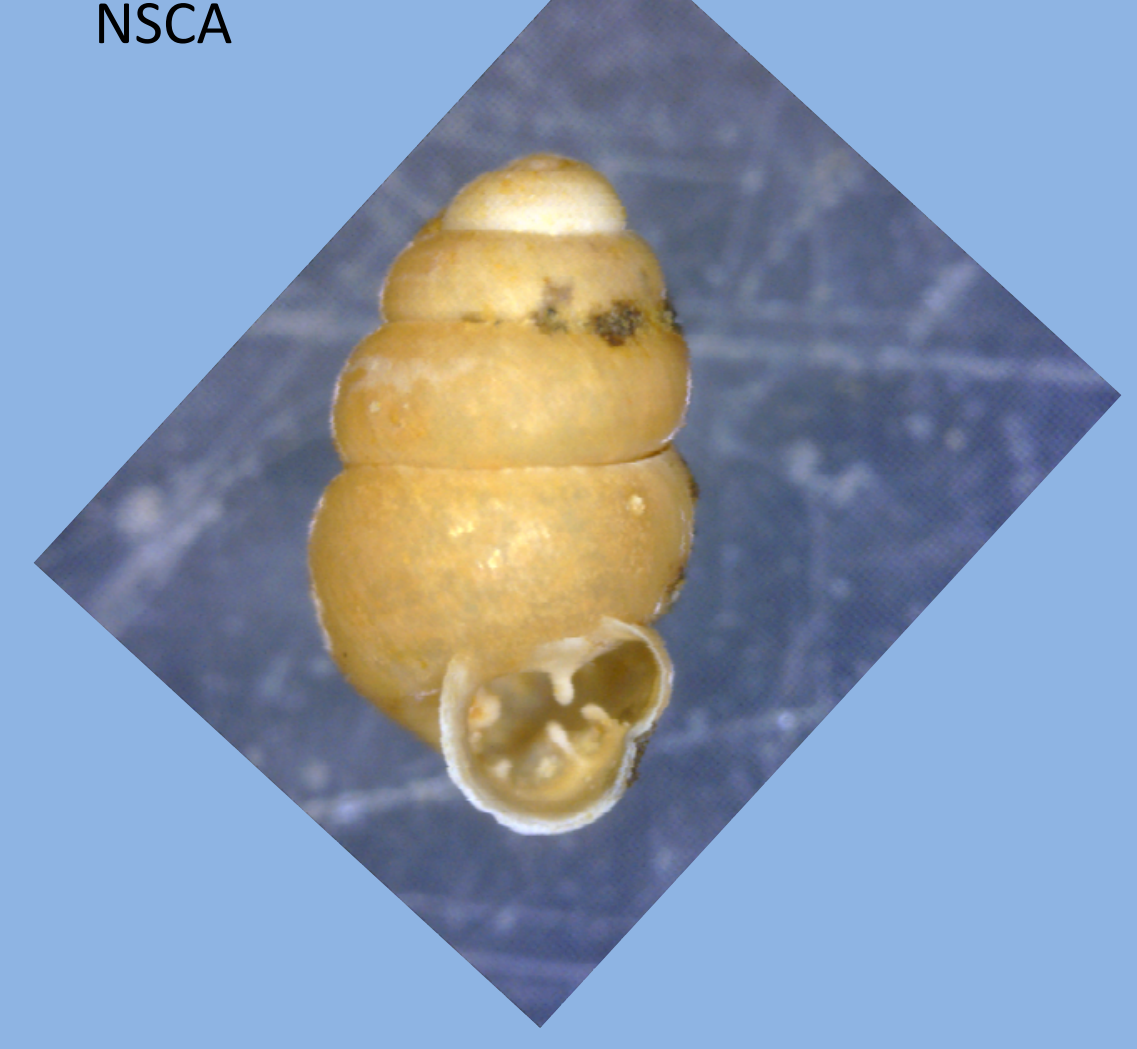

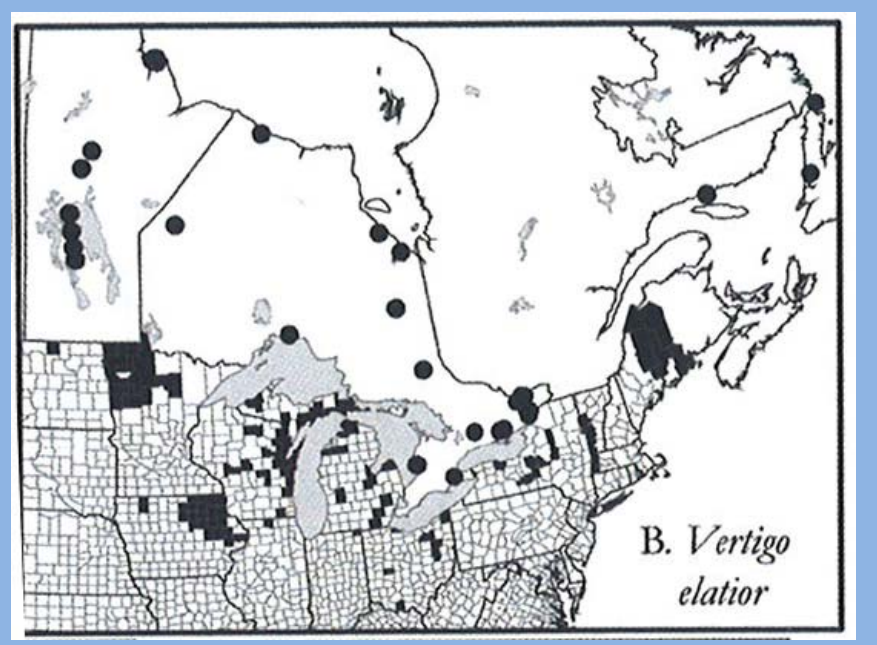

Individuals occur in well-decomposed humid leaf litter in a variety of open and wooded wetland habitats, including wet prairie, fens, wet meadows, tundra, and black ash, tamarack, and black spruce swamp forests.

Nekola and Coles, 2010

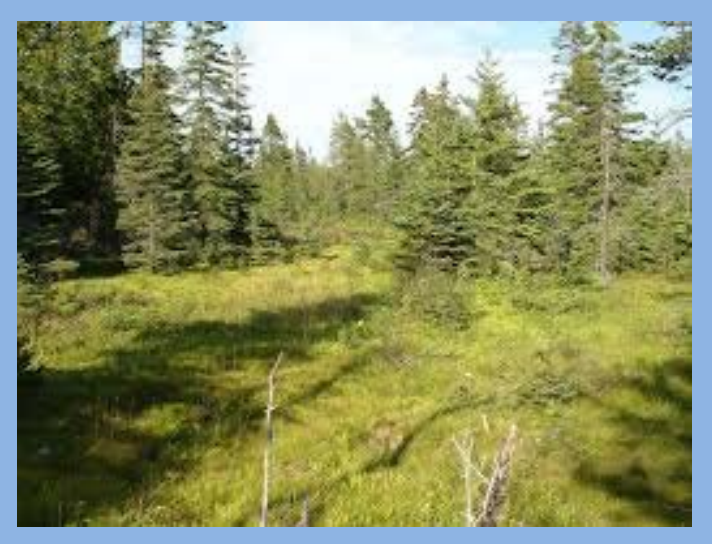




\section{Overlapping Range Method: overlap of modern distributions}
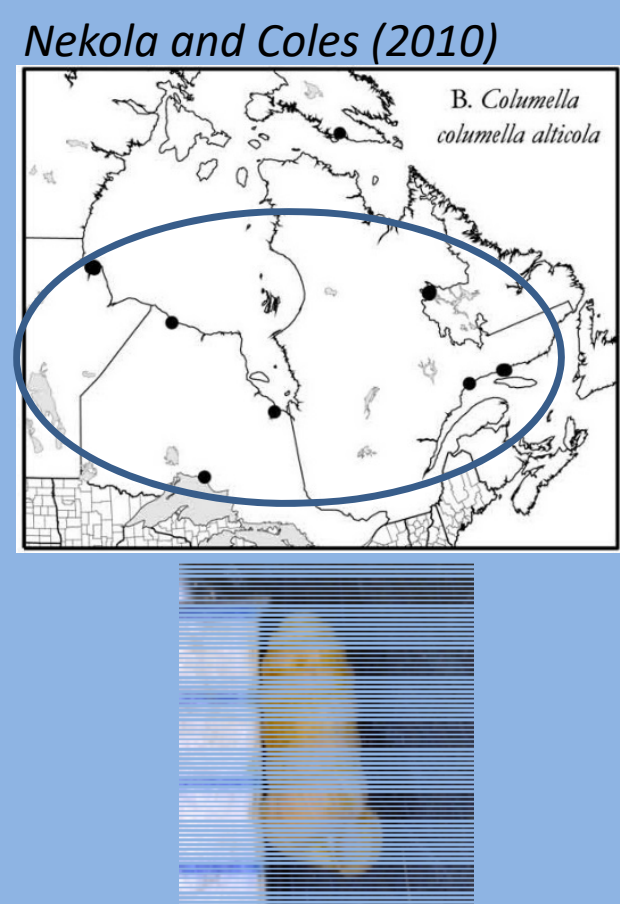

Columella alticola

\section{Nekola and Coles (2010)}
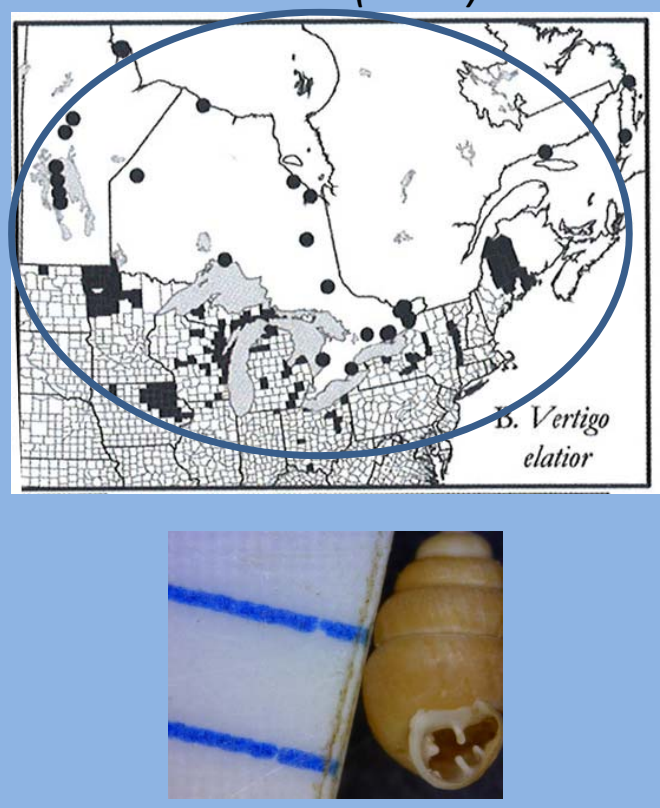

Vertigo eliator
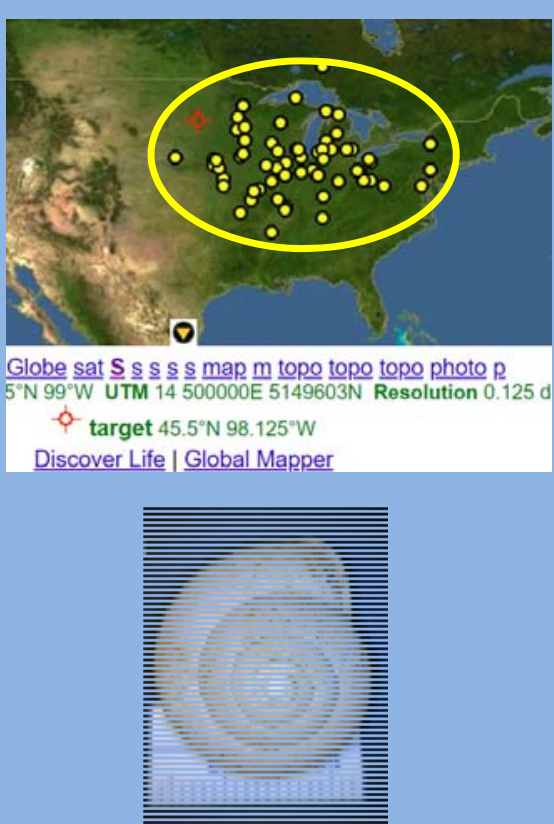

Webbhelix multilineata

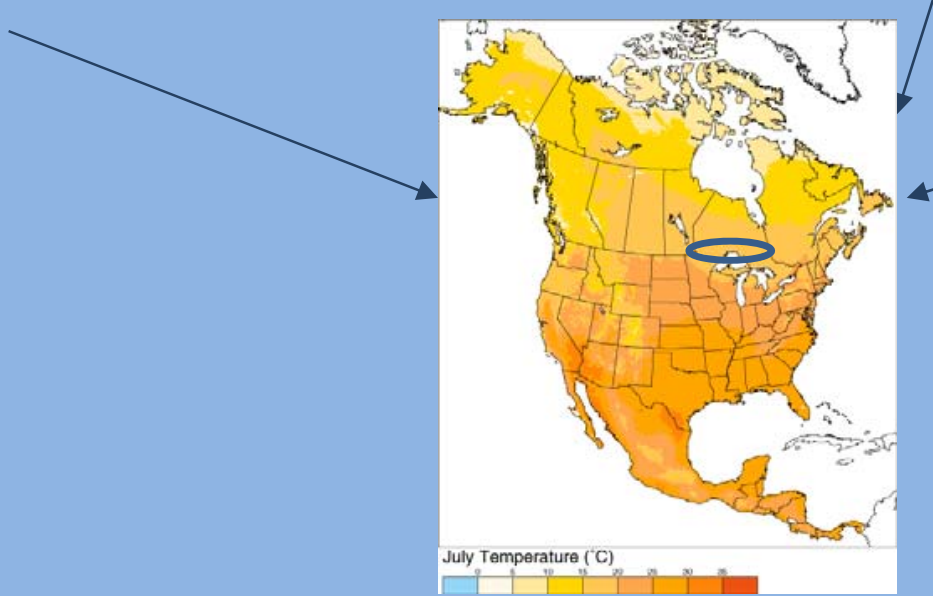

approx. mean July temperature: $\sim 17^{\circ}$ to $18^{\circ} \mathrm{C}$ 


\section{Mutual Climatic Range Method: obtaining climatic data for coldest and warmest modern location by species}

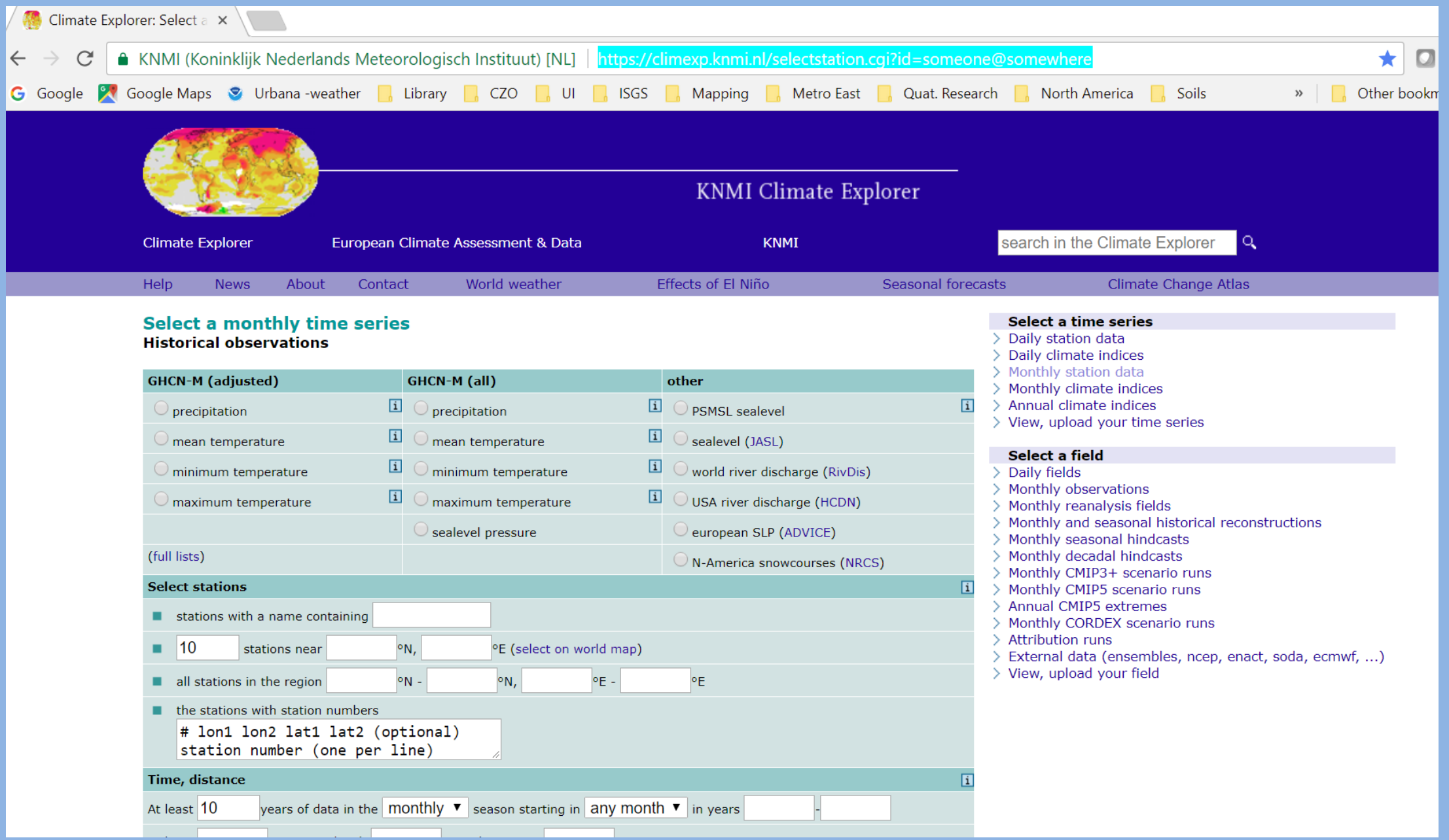

https://climexp.knmi.nl/selectstation.cgi?id=someone@somewhere 


\section{Mutual Climatic Range Method (non-analog result); Illinois Valley loess}

( 39.5 to $40^{\circ} \mathrm{N}$ lat; $\sim 25$ to $20 \mathrm{cal} \mathrm{ka)}$

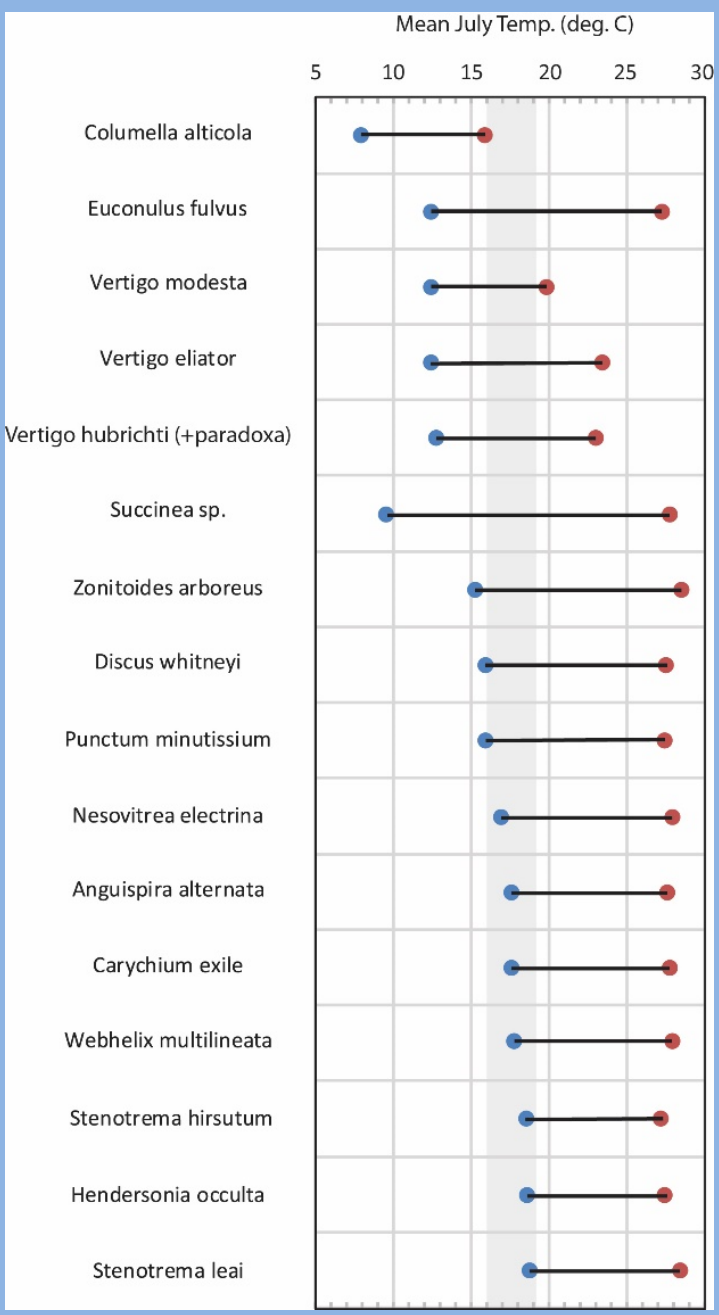

- mean July temp 16 to $19{ }^{\circ} \mathrm{C}$

- $\mathrm{LGM} \sim 5$ to $8{ }^{\circ} \mathrm{C}$ cooler than today $\left[24^{\circ} \mathrm{C}\right]$

- non-analog condition (MCR would be 18 to $20^{\circ} \mathrm{C}$ if Columella alticola not present)

- either C. alticola or other species existed beyond their present-day climatic range (do not coexist together today) 


\section{Demazenod Section; Mississippi Valley loess}

( $38.5^{\circ} \mathrm{N}$ lat; St. Louis area; 22 to $20 \mathrm{cal} \mathrm{ka)}$
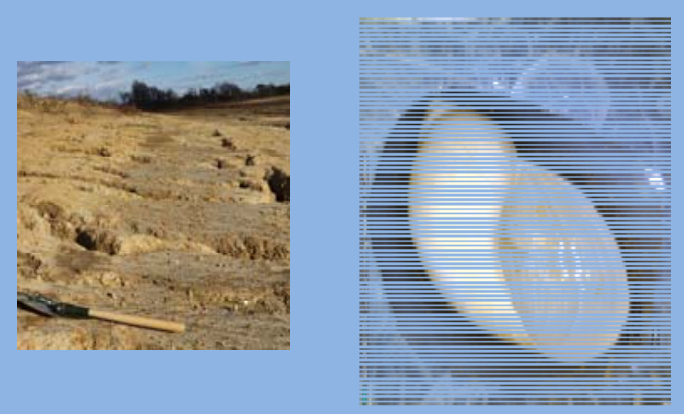

20,200 cal yrs 21,900 cal yrs

ther +4

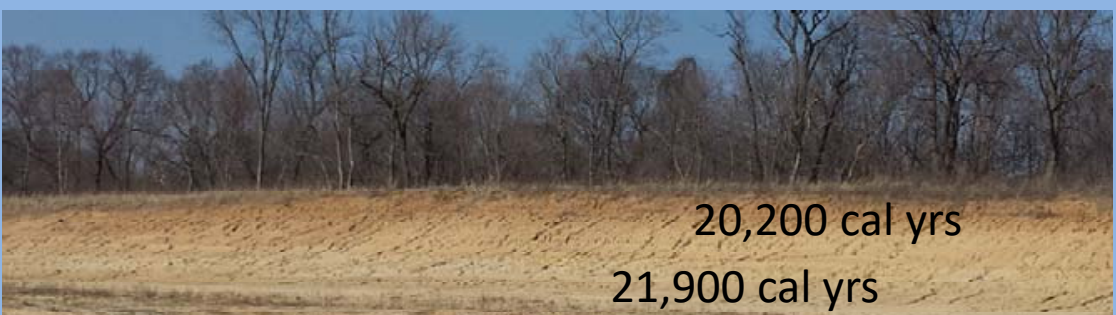

Demazenod Section; Belleville, IL

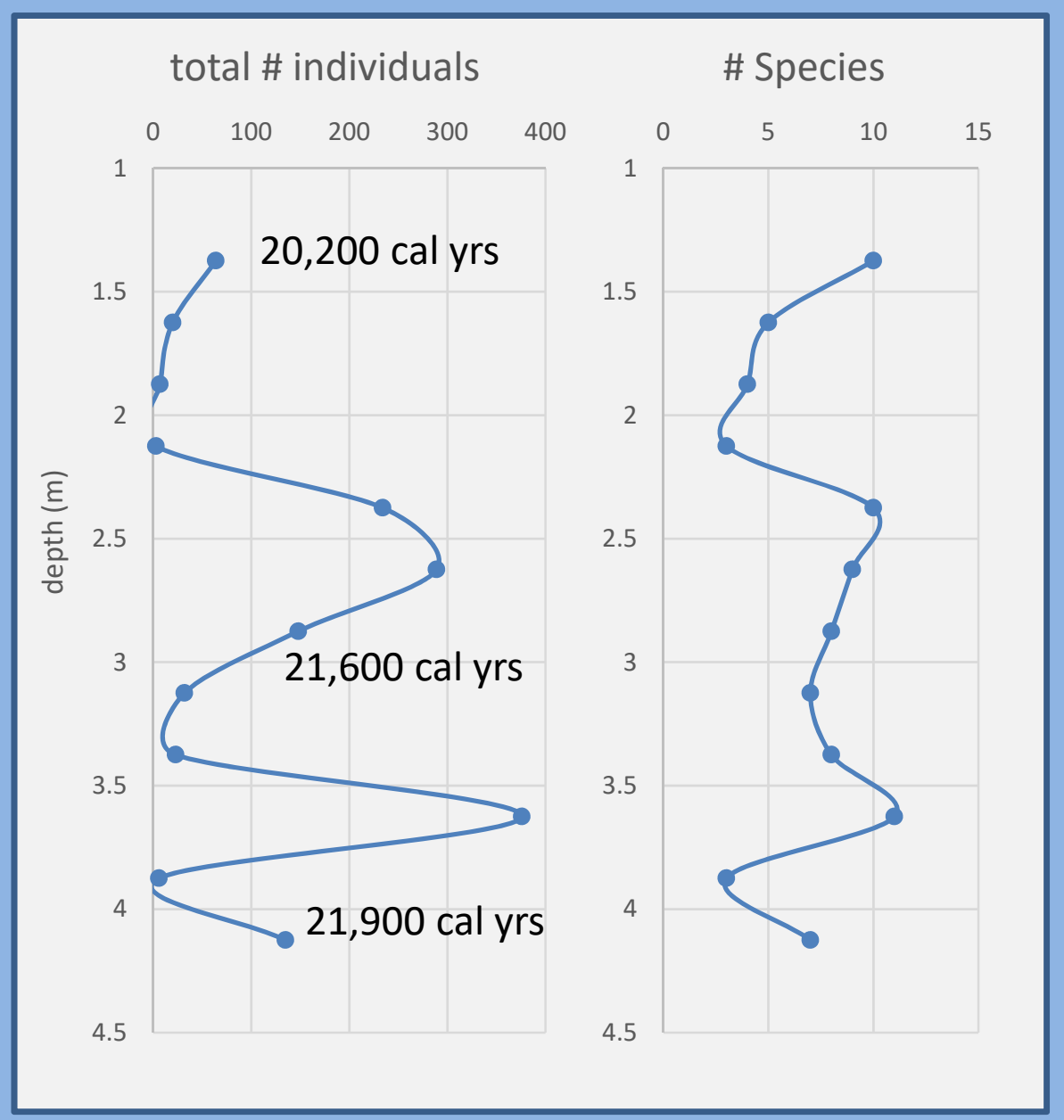

Demazenod Section; MCR 


\section{MCR Figure; Demazenod Section (mid-Mississippi Valley)}

( $38.5^{\circ} \mathrm{N}$ lat; St. Louis area; 22 to 20 cal ka)

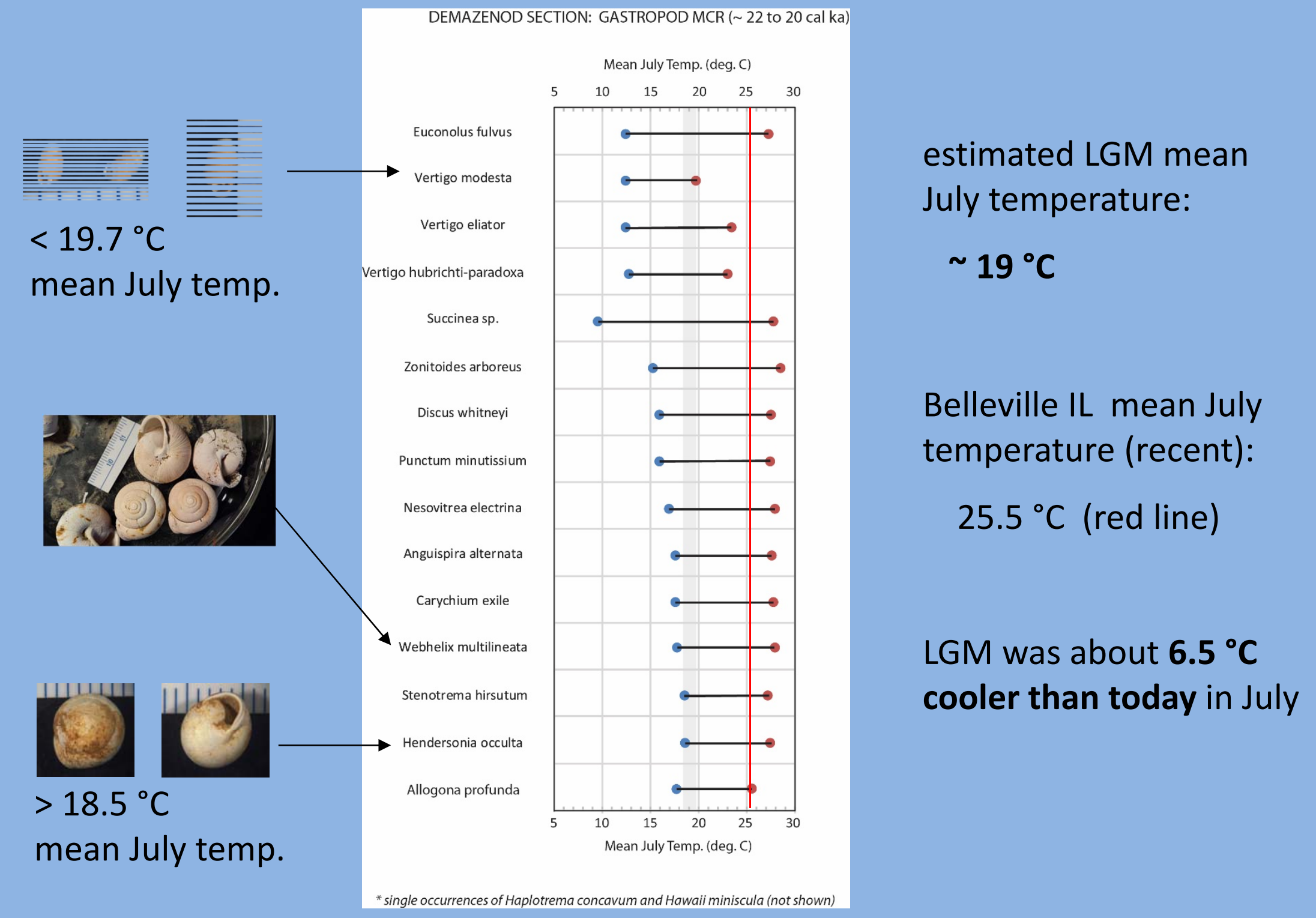




\section{Clayton Section; central Indiana}

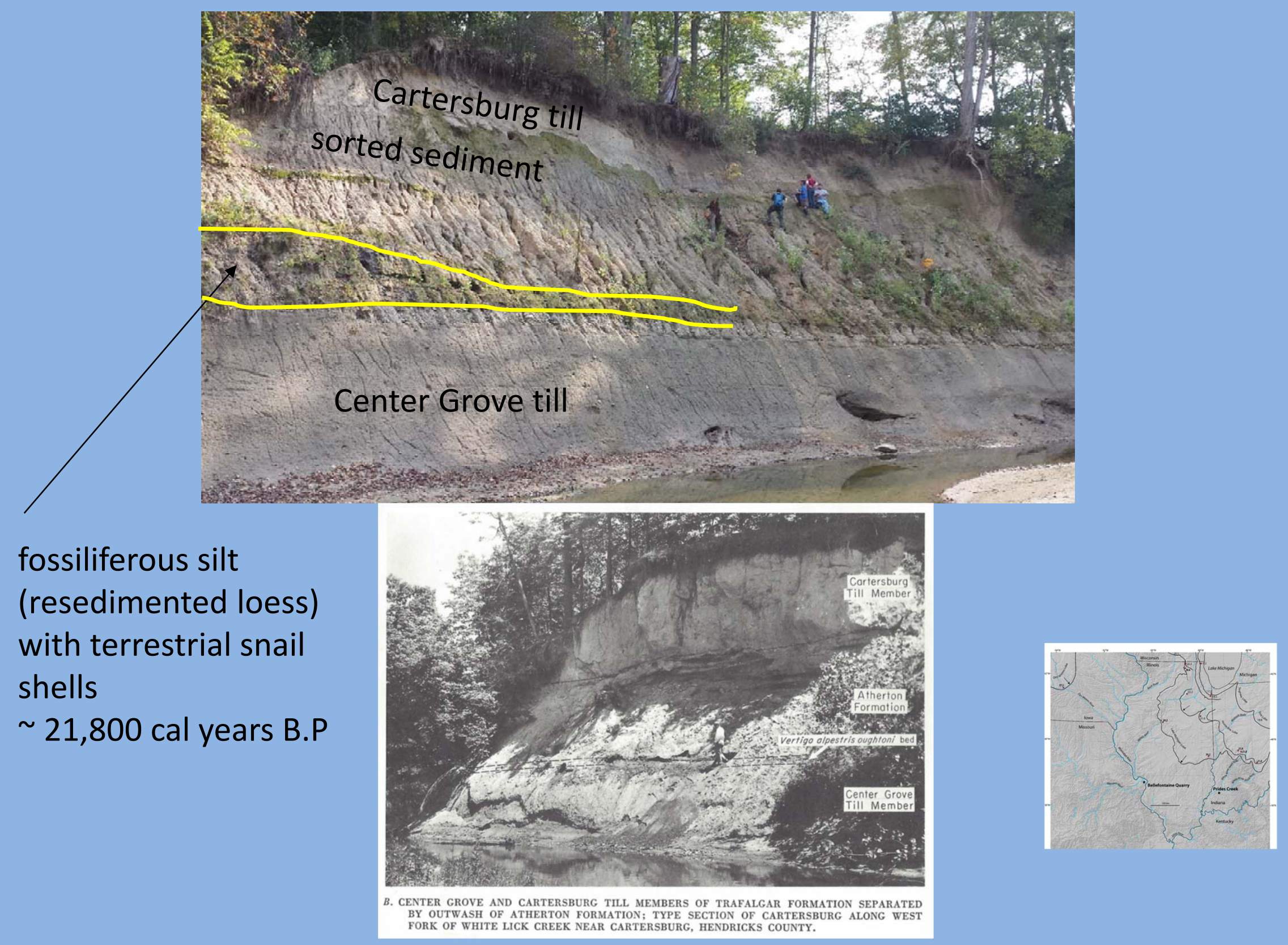




\section{Terrestrial Gastropod Assemblage: Clayton and Plainfield Sections}

\section{CLAYTON SECTION, INDIANA \\ fauna found between two \\ Wisconsin Episode tills}

Sample Interval (above base of

silt unit; (and gravel lag on till)

80-85 cm (upper)

$65-80 \mathrm{~cm}$ (middle)

60-65 cm (lower)

Total

Comments another 3 meters to till above (ow \& silt in

$$
\text { between) }
$$

\section{Columella Columella}

\section{4}

17

16

1

41

17
whitney

Euconulus

Succineidae

Pupilla

Pupilla

Vallonia gracilicosta

\section{Vertigo}

modesta Vertigo oughtoni juveniles Total

\begin{tabular}{l}
34 \\
5 \\
3 \\
42 \\
\hline
\end{tabular}
$15 \quad 110$
$6 \quad 144$
$0 \quad 54$
21
308
small; shell shape
similar to
Catinella avara

\begin{tabular}{|c|c|}
\hline 29 \\
\hline 21 \\
\hline 4 \\
\hline 54 \\
\hline hape \\
\hline $\begin{array}{c}\text { formerly Pupilla } \\
\text { muscorum; } \\
\text { Nekola et al., } \\
2014\end{array}$ \\
\hline
\end{tabular}

11

33

75

$36-108$

492

$17 \quad 12$

62

\begin{tabular}{l|l|l}
\hline 0 & 1 & 7 \\
\hline
\end{tabular}

7

\begin{tabular}{l}
$28 \quad 46$ \\
\hline
\end{tabular}

144

\begin{tabular}{|c|c|}
\hline 144 & 13 \\
\hline 20 & 27 \\
\hline 200 & 148 \\
\hline $\begin{array}{c}\text { V. genesioides is } \\
\text { new species of } \\
\text { Nekola; upcoming } \\
\text { paper }\end{array}$ & $\begin{array}{c}\text { juveniles of } \\
\text { Vertigo, } \\
\text { Discus, } \\
\text { Sucinea, and } \\
\text { Pupilla }\end{array}$ \\
\hline
\end{tabular}

441

117

1050

\section{PLAINFIELD SECTION, INDIANA \\ fauna found between two Wisconsin Episode \\ tills; collected by Henry Loope; ids by David Grimley}

\begin{tabular}{|c|c|c|c|c|c|c|c|c|c|}
\hline Sample Interval & $\begin{array}{l}\text { Columella } \\
\text { alticola }\end{array}$ & $\begin{array}{c}\text { Discus } \\
\text { whitneyi }\end{array}$ & $\begin{array}{l}\text { Euconulus } \\
\text { fulvus }\end{array}$ & $\begin{array}{c}\text { Succineidae } \\
\text { (cf. Catinella avara) }\end{array}$ & $\begin{array}{c}\text { Pupilla } \\
\text { hudsonianum }\end{array}$ & $\begin{array}{c}\text { Vallonia } \\
\text { gracilicosta }\end{array}$ & $\begin{array}{l}\text { Vertigo } \\
\text { modesta }\end{array}$ & $\begin{array}{l}\text { (?) Vertigo } \\
\text { genesioides } \\
\text { or oughtoni }\end{array}$ & $\begin{array}{c}\text { Total } \\
\text { Individuals }\end{array}$ \\
\hline typical size & & & & 2 to $6 \mathrm{~mm}$ & $3 \mathrm{~mm}$ & & $2.5 \mathrm{~mm}$ & $2 \mathrm{~mm}$ & \\
\hline $0-10 \mathrm{~cm}$ below till & 0 & 0 & 0 & 79 & 10 & 0 & 4 & 4 & 97 \\
\hline
\end{tabular}

\section{* boreal forest to mixed boreal tundra environment}




\section{MCR Figure; central Indiana silts (loess-derived)}

( 39.4 to $39.7^{\circ} \mathrm{N}$ latitude; Indianapolis area)

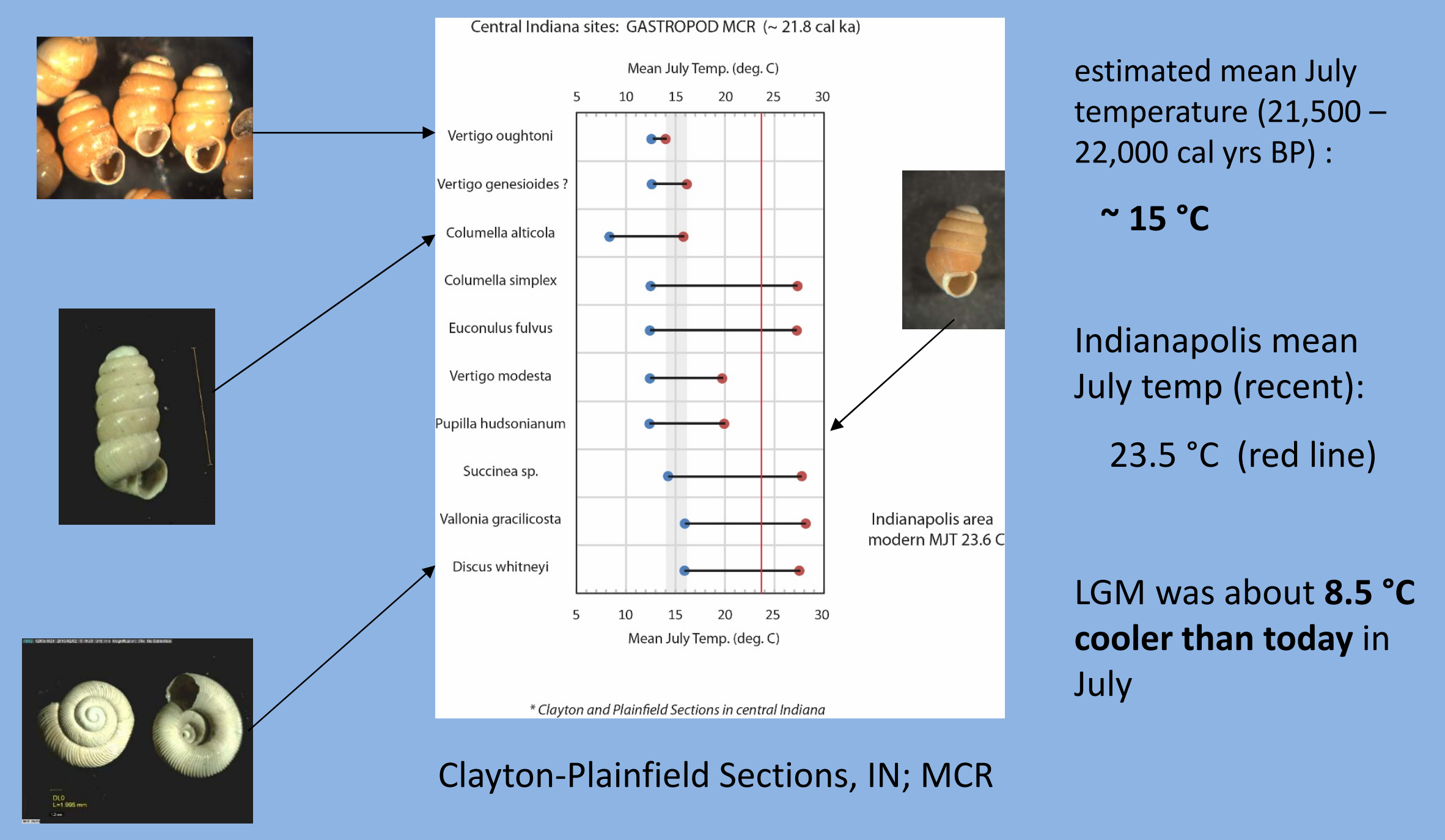


Climatic and ecological gradient: 22,000 to 21,000 years BP

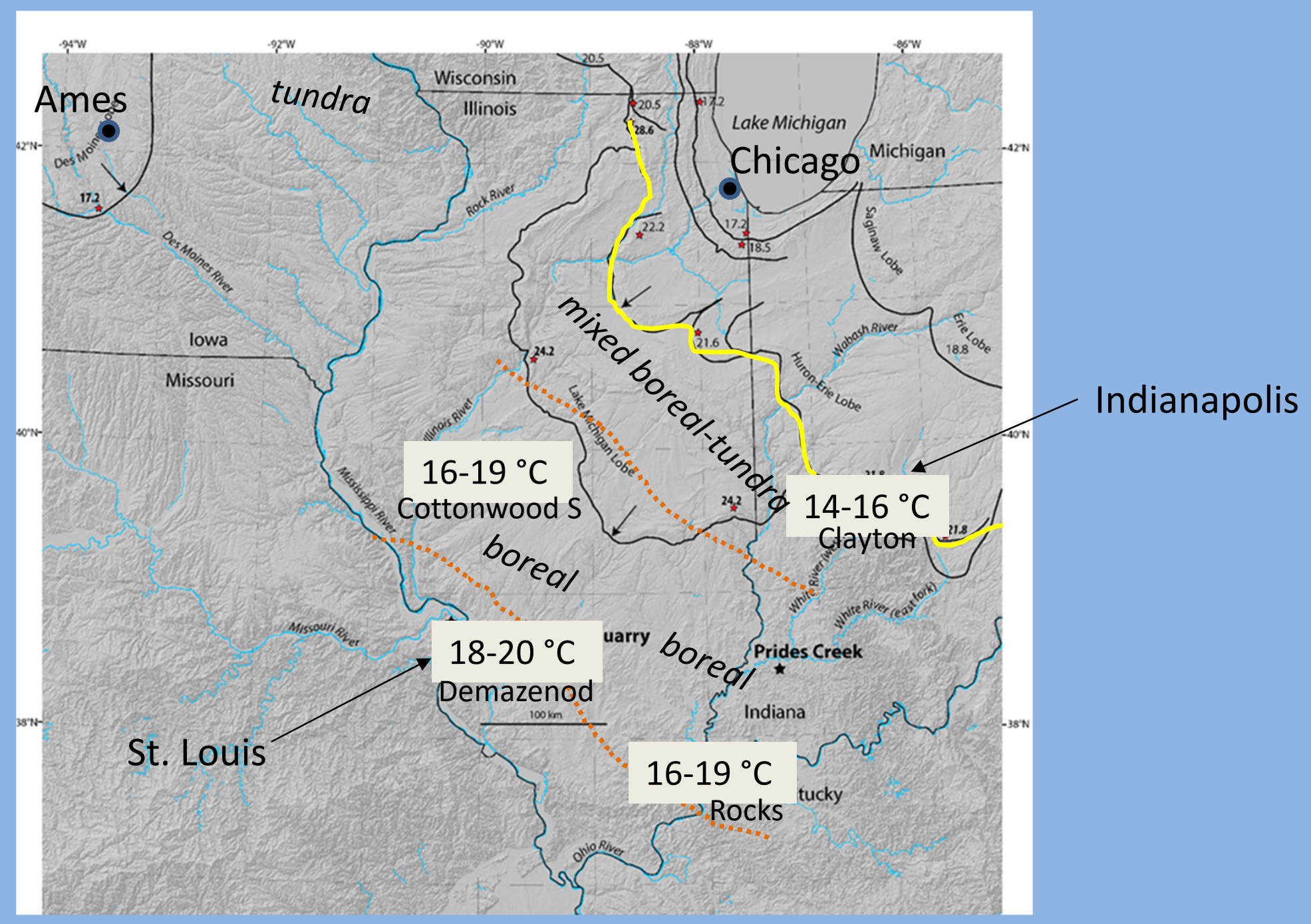

(Rocks Section data from 24,500 cal ka) 
Pollen-based last glacial vegetation map of North America

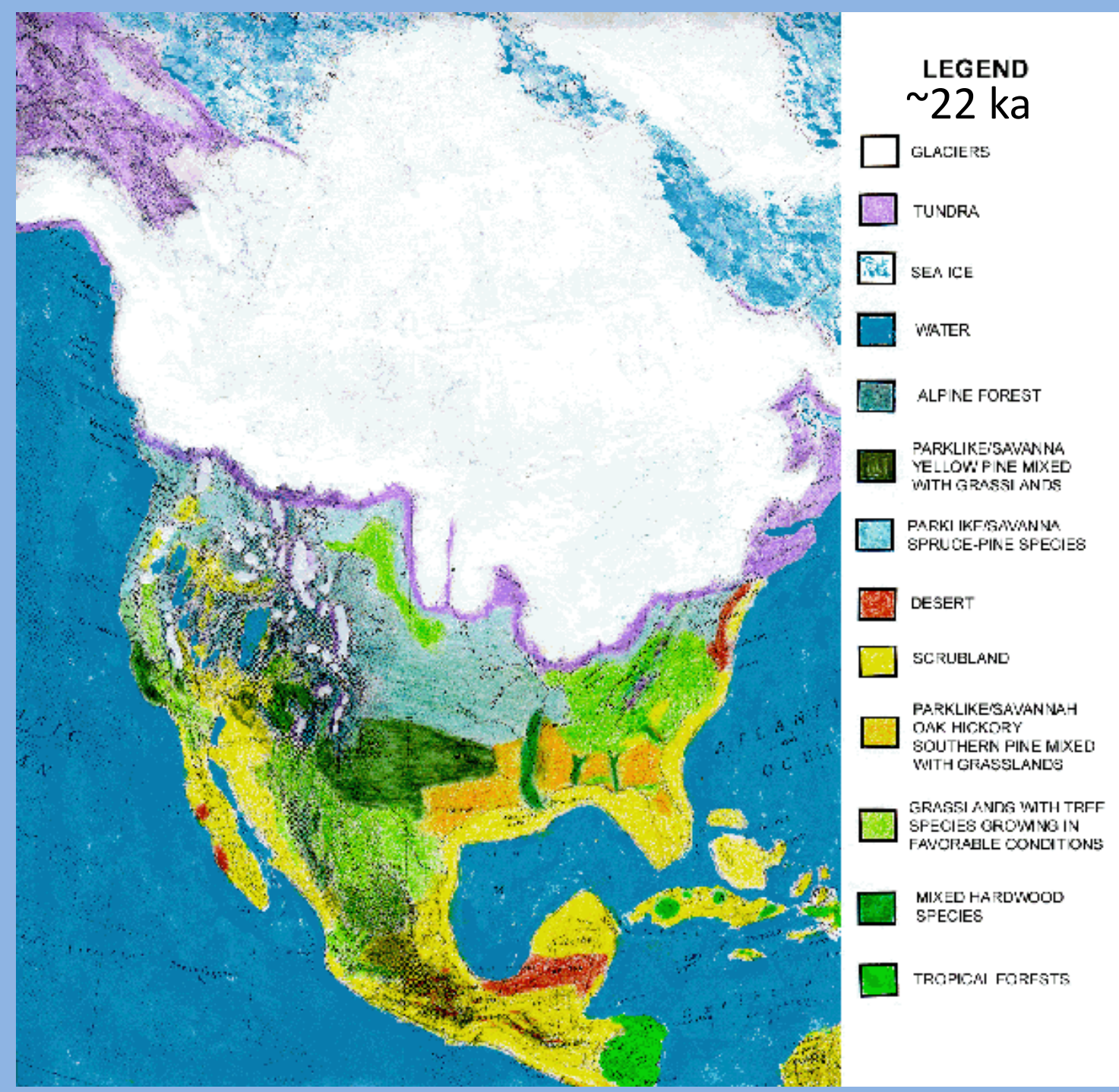

https://www.srs.fs.fed.us/sustain/report/histry/histry-31.htm (modified from Delcourt and Delcourt, 1985) 


\section{Rocks Section (Peoria loess)}

\section{( 37.7 deg. N lat; western Kentucky)}
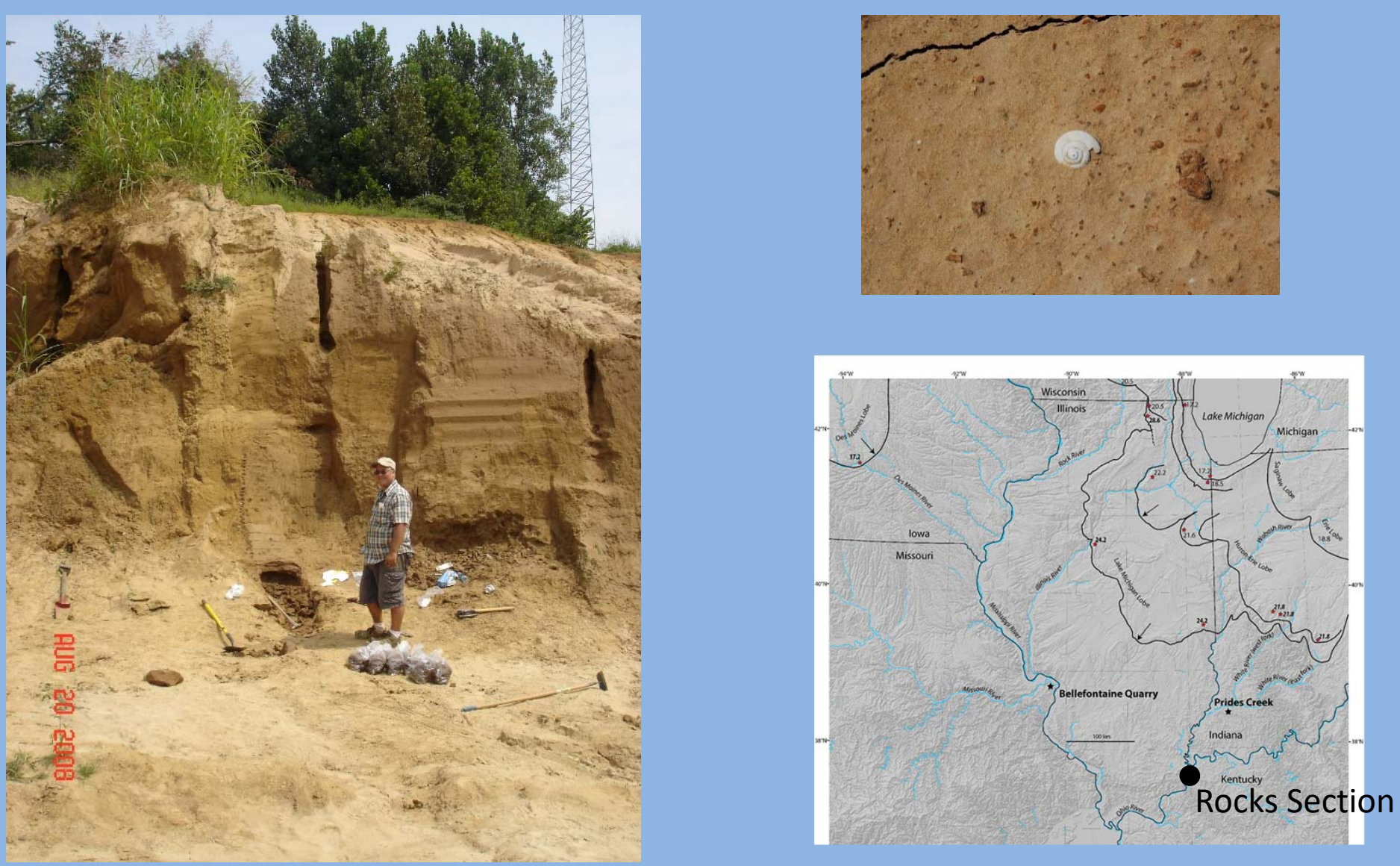


\section{MCR; Rocks Section loess ( 25 cal ka)}

\section{( 37.7 deg. N lat; western Kentucky)}

\section{8 to $2.8 \mathrm{~m}$ depth ( $25 \mathrm{cal} \mathrm{ka)}$}

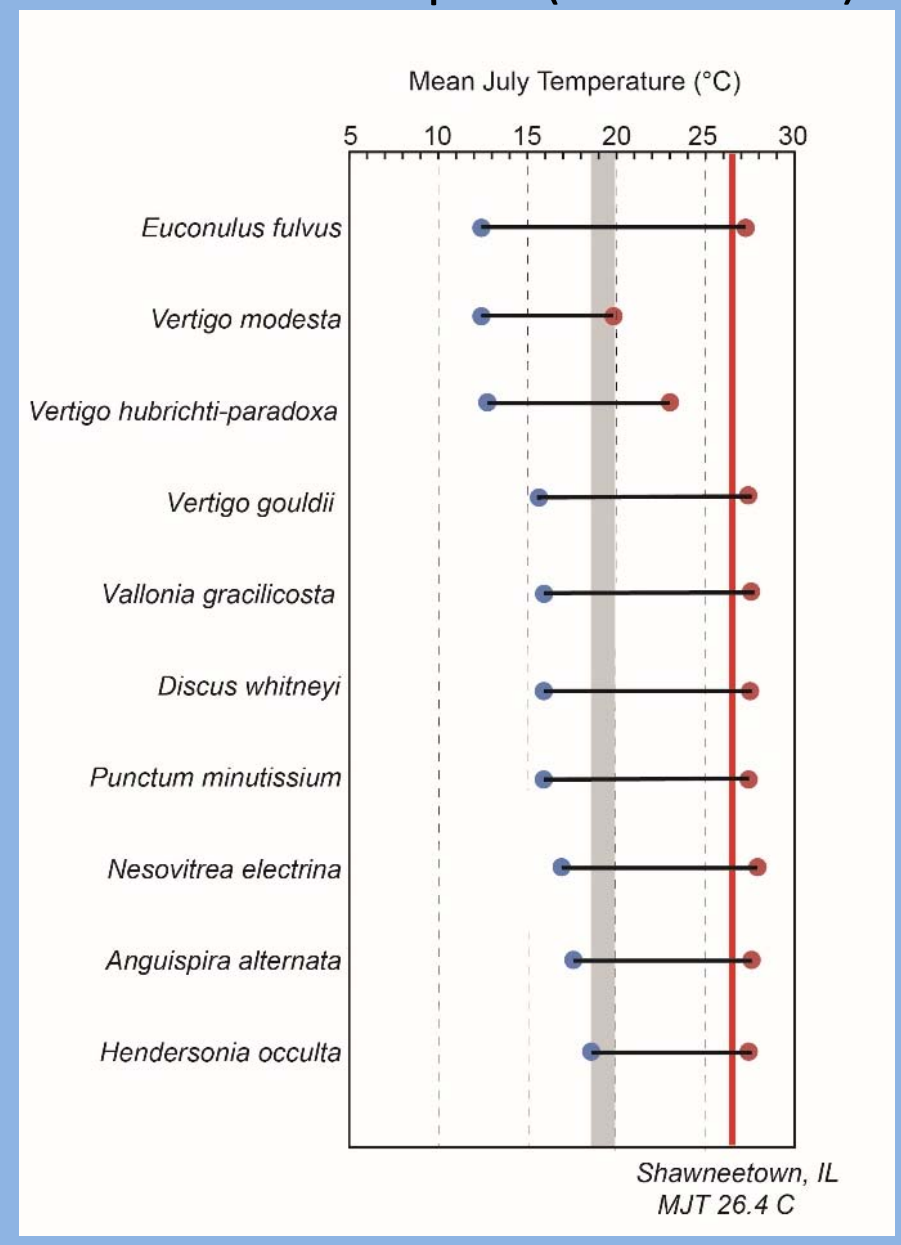

- MJT estimate: $19^{\circ} \mathrm{C}$

$\sim 24,500$ to 25,500 cal yrs ago (LGM)

- about $7^{\circ} \mathrm{C}$ cooler MJT than today $\left(26^{\circ} \mathrm{C}\right)$

Rocks Section; western KY.........near Shawneetown, IL 


\section{MCR; Rocks Section loess ( 26 cal ka)}

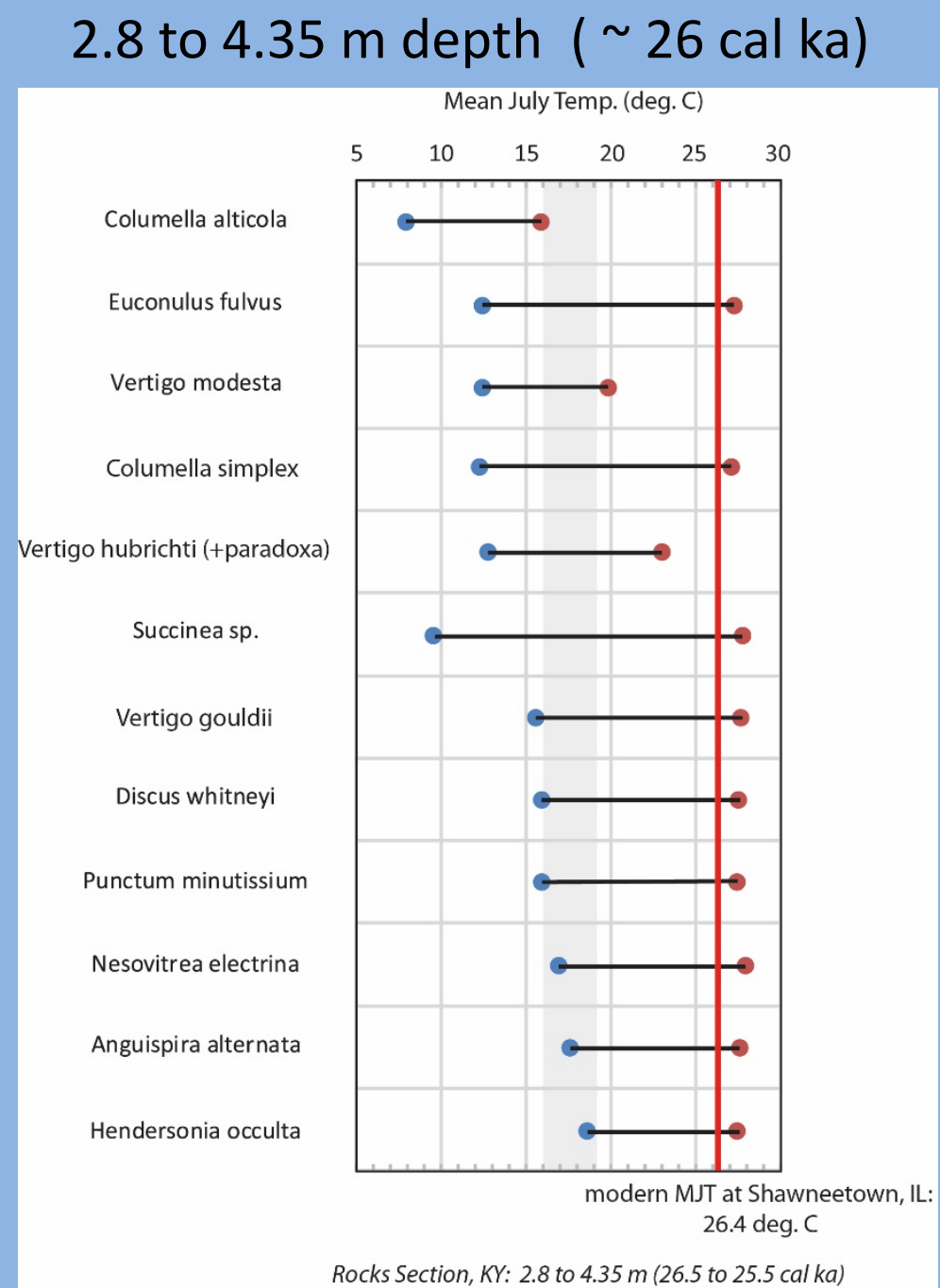

- MJT estimate: 16 to $19{ }^{\circ} \mathrm{C}$

$\sim 25,500$ to 26,500 cal yrs ago (LGM)

- about 7 to $10^{\circ} \mathrm{C}$ cooler July temperature than today $\left(26^{\circ} \mathrm{C}\right)$ 


\section{MCR; Rocks Section loess ( 28 to 29 cal ka)}

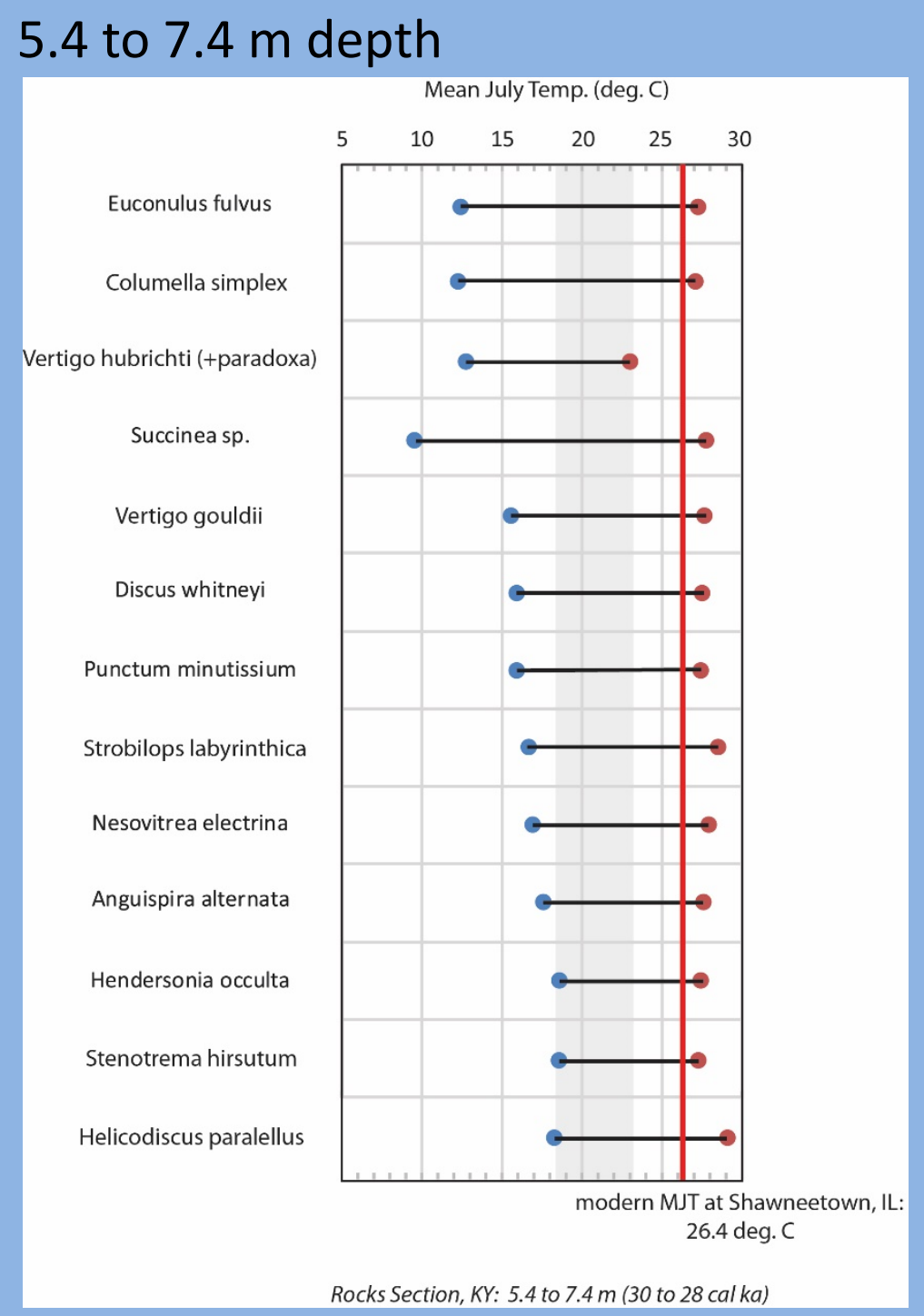

- MJT estimate: $\mathbf{1 8 . 5}$ to $23^{\circ} \mathrm{C}$

$\sim 27,500$ to 29,500 cal yrs ago (LGM)

- about 3 to $8^{\circ} \mathrm{C}$ cooler July temperature than today $\left(26^{\circ} \mathrm{C}\right)$ 


\section{MCR; Rocks Section loess ( 30 cal ka)}

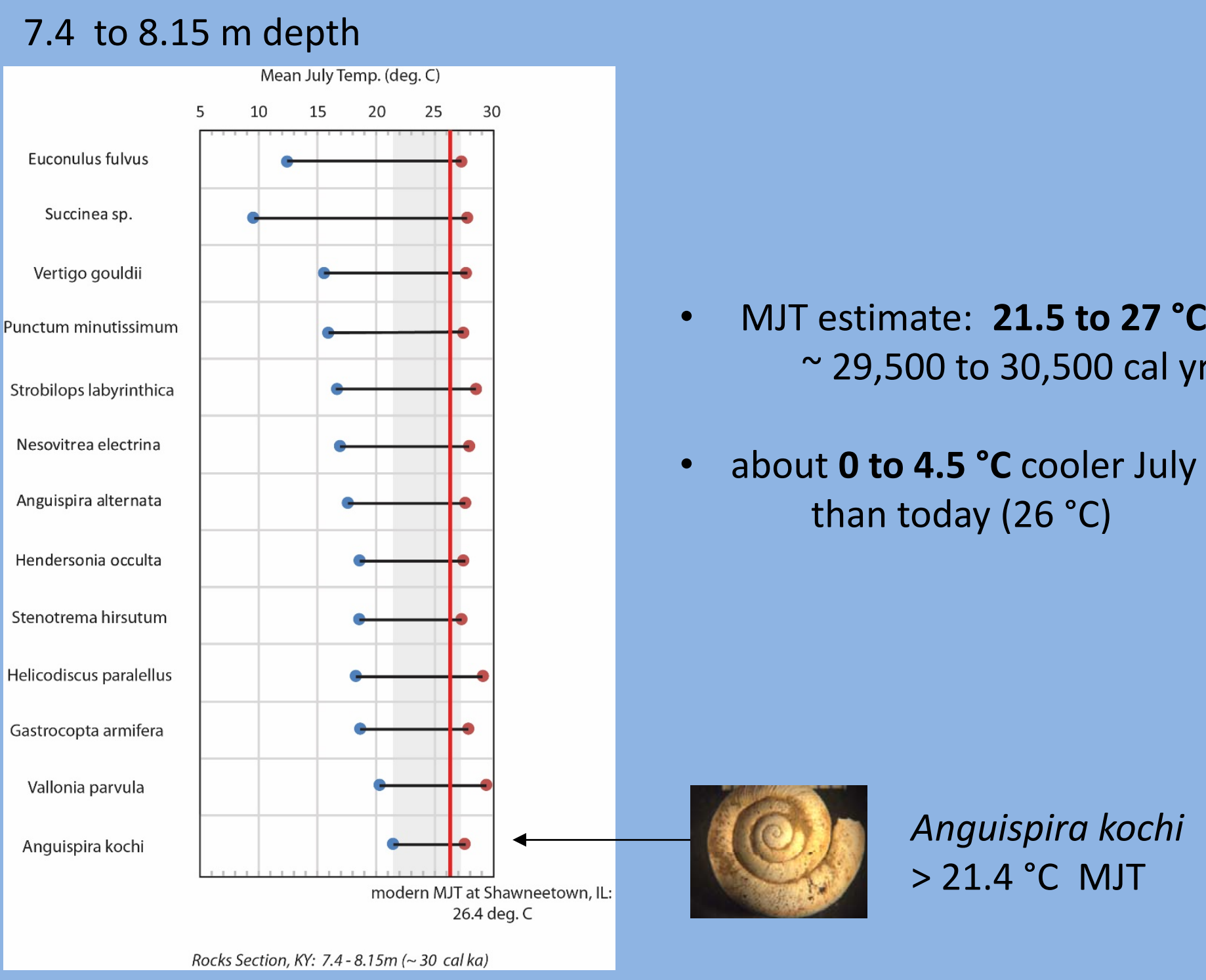


Rocks Section (western Kentucky) paleoclimate history (30 to 24 cal ka)

Peoria-

Roxana Silt transition

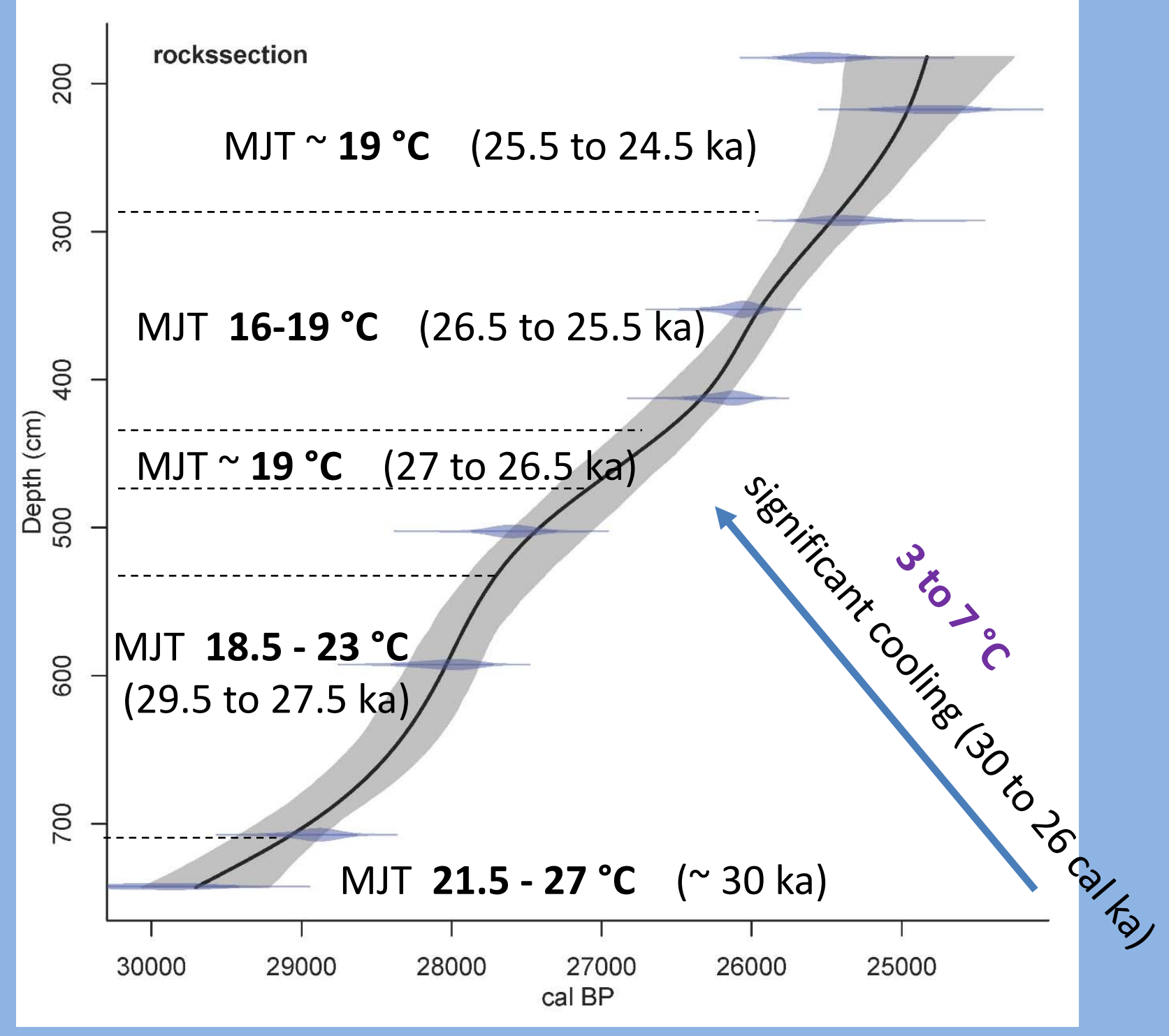




\section{Summary}

- overall LGM paleoclimate ( 22 to $21 \mathrm{cal} \mathrm{ka):}$

MJT $15^{\circ} \mathrm{C}$ (Indianapolis) to $\sim 19{ }^{\circ} \mathrm{C}$ (St. Louis)

today $23.5^{\circ} \mathrm{C} \quad 25.5^{\circ} \mathrm{C}$

- 6 to $9^{\circ} \mathrm{C}$ cooler MJT during LGM than today

$>$ regional climatic gradient (NE-SW) about double that of today

$>$ likely relates to proximity of glacial margin

- boreal (StL) to boreal-tundra (Indy) ecosystem (based on snails, plant macrofossils)

- 3 to $7^{\circ} \mathrm{C} \mathrm{MJT} \mathrm{cooling} \mathrm{during} \mathrm{approach} \mathrm{to} \mathrm{LGM} \mathrm{(30} \mathrm{to} 26$ cal ka)

(based on terrestrial gastropod mutual climatic range method)

- more studies needed along other Midwest valley segments (project ongoing!) 
- High resolution chronologies from terrestrial gastropods in last glacial loess sequences in the midcontinental USA (Peoria Silt; $30-16 \mathrm{cal} \mathrm{ka)} \mathrm{provide} \mathrm{a} \mathrm{means} \mathrm{to} \mathrm{evaluate} \mathrm{temporal} \mathrm{and} \mathrm{spatial}$ variations in sedimentation rates, climate, and environments. From a loess-paleosol section in western Kentucky, stratigraphic changes in gastropod assemblages imply climatic cooling at 29 ka and again at $27 \mathrm{ka}$, leading into the last glacial maximum. At $30 \mathrm{ka}$, a temperate summer climate $\left(\mathrm{MJT}>21^{\circ} \mathrm{C}\right)$ and forested ecosystem is implied by a diverse assemblage (15 species) that includes Anguispira kochi-Gastrocopta pentodon-Helicodiscus parallelus. At 29-27 ka, the assemblage includes Discus whitneyi, Hendersonia occulta and $H$. parellelus, but lacks $A$. kochi and Gastrocopta. After $27 \mathrm{ka}$, the introduction of Columella alticola, Vertigo modesta, and Vallonia gracilicosta and disappearance of Helicodiscus records a shift to substantially colder (MJT 15-19 ${ }^{\circ} \mathrm{C}$ ), boreal conditions. Finer-scale variability in species composition, sedimentation rates, and weak paleosol development may record multi-centennial fluctuations in climate and/or glacial lobe margins.

- A strong NE-SW regional climatic gradient at 22-21 cal ka (during last glacial maximum) is suggested by contrasting gastropod assemblages from central Indiana to southwestern Illinois. Resedimented loess between till units in central Indiana (Clayton and Plainfield Sections) contains abundant cold tolerant species Vertigo modesta-V. oughtoni-Columella alticola, reflecting boreal to borderline tundra conditions near the ice margin. At the same latitude $\left(40^{\circ} \mathrm{N}\right)$ in loess along the Illinois Valley, the assemblage transitions to a more diverse one, with only rare V. modesta and $C$. alticola and more southern species. In southwestern Illinois (St. Clair County), the loess fauna has few cold-climate species but contains W. multilineata, Allogona profunda, and Stenotrema hirsutum, suggesting more temperate conditions. We thus envision a steep paleoclimatic gradient from present-day Indianapolis (MJT $14-16^{\circ} \mathrm{C}$ ) to St. Louis, Missouri (MJT $18-20^{\circ} \mathrm{C}$ ). These interpretations are complementary to plant macrofossil and regional pollen records, but enable a more detailed characterization of environmental change across the central USA during the last glacial maximum. 